\title{
$B$-physics studies at LHC with the ATLAS detector
}

\section{loannis Nomidis*†}

Aristotle University of Thessaloniki

E-mail: ioannis.nomidis@cern.ch

\begin{abstract}
Measuring heavy flavour production at LHC provides an opportunity for a new insight into QCD at the new center-of-mass energy of $7 \mathrm{TeV}$. ATLAS results for quarkonium production are presented and compared to theoretical predictions of various QCD models. Open $b$ and $b \bar{b}$ cross section measurements are compared to next-to-leading order calculations. ATLAS capability to reconstruct $B$-hadrons in exclusive decays is reported, as well as measurements of $B$-hadron lifetimes.
\end{abstract}

LHC on the March,

November 16-18, 2011

Protvino, Moscow region, Russian Federation

\footnotetext{
*Speaker.

${ }^{\dagger}$ On behalf of the ATLAS Collaboration
} 


\section{Introduction}

ATLAS $B$-physics program concentrates on a list of measurements that are of particular interest in understanding QCD and on measurements of $\mathrm{CP}$ violation and searches of rare decays that will enlarge the discovery potential of the experiment. In ATLAS, the production of $b$-quark in $p-p$ collisions is studied through measurement of charmonium and bottomonium production via direct observation of $J / \psi$ and $\Upsilon$. Results are also presented for open $b$ and $b \bar{b}$ production, measured via identification of $b$-jets.

Besides the measurement of production cross sections, ATLAS provides results on the $b$ properties such as lifetimes of $B$-hadrons measured from fully reconstructed exclusive decays. The ability of the ATLAS detector to reconstruct exclusive $B$-decays is demonstrated by measuring the lifetimes of $B_{d}^{0}$ and $B_{s}^{0}$ with early data. Such studies are mandatory for interesting measurements like that for the lifetime of $\Lambda_{b}$ and CP-violation in $B_{s}^{0} \rightarrow J / \psi \phi$.

\section{Experimental issues}

\subsection{LHC Performance}

Results discussed in these proceedings were obtained using data taken during the 2010 LHC operation at a center-of-mass energy of $7 \mathrm{TeV}$ for $p$ - $p$ collisions, with a peak luminosity of $2 \cdot 10^{32} \mathrm{~cm}^{-2} \mathrm{~s}^{-1}$. The total integrated luminosisity of the 2010 dataset is $45 \mathrm{pb}^{-1}$ with a systematic uncertainty of 3.4\% [1]. By the time of this conference, November 2011, the LHC's $p-p$ run for 2011 had been already completed, delivering to the experiments more than $5 \mathrm{fb}^{-1}$ and the peak luminosity reaching $3.65 \cdot 10^{33} \mathrm{~cm}^{-2} \mathrm{~s}^{-1}$. ATLAS recorded data from runs in 2010 and 2011 with $95 \%$ efficiency. The estimated the total integrated luminosity for the 2011 dataset is $5.2 \mathrm{fb}^{-1}$ with a preliminary systematic uncertainty of $3.9 \%$.

\subsection{Trigger strategy}

$B$-physics studies in ATLAS are mainly based on muon triggers. In the low-luminosity datataking periods single muon triggers are used. As luminosity increases, these triggers are either prescaled or increasing thresholds in $p_{T}$. Neither of these choices is good for $B$-physics, so in order to be able to reconstruct $B$-hadrons of low- $p_{T}$, dedicated High-Level di-muon Triggers (HLT) were used, based on single or di-muon hardware triggers from the muon spectrometer. Using dedicated algorithms at HLT to identify di-muon decays, ATLAS can trigger on quarkonia, on $B$-hadrons decaying to $J / \psi+X$ and on rare muonic $B$-decays like $B_{s}^{0} \rightarrow \mu^{+} \mu^{-}$.

\section{Charm and beauty production at hadron colliders}

LHC provides the chance to test calculations of pertubative QCD for quarkonium and $b$-production at a new energy regime, higher transverse momenta and in wider rapidity range than before, thanks to the good coverage of the detectors of the LHC experiments. The motivation to study quarkonium production at LHC is strong. From the theoretical point of view, the production mechanisms of quarkonium states like $J / \psi$ and $\Upsilon$ are not clearly understood and in addition to that, the Tevatron experiments have not provided consistent or conclusive results. Thus, new cross section and spin 


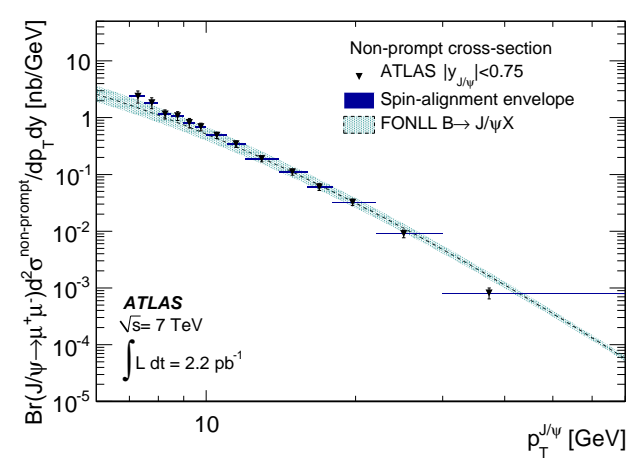

(a) $|y|<0.75$

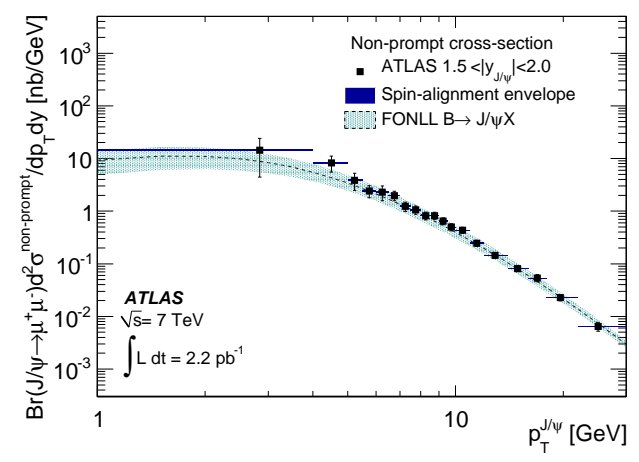

(c) $1.5<|y|<2$

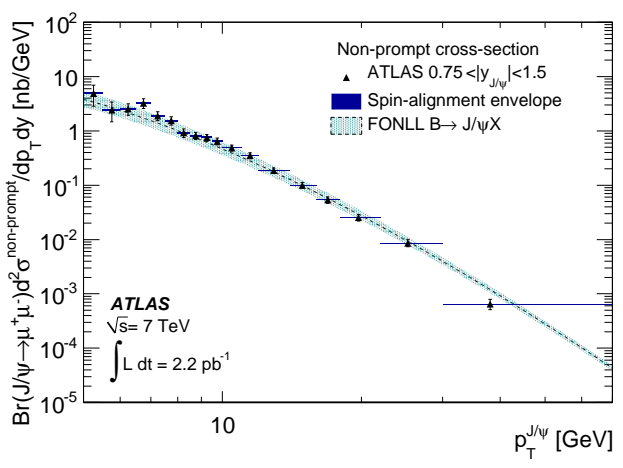

(b) $0.75<|y|<1.5$

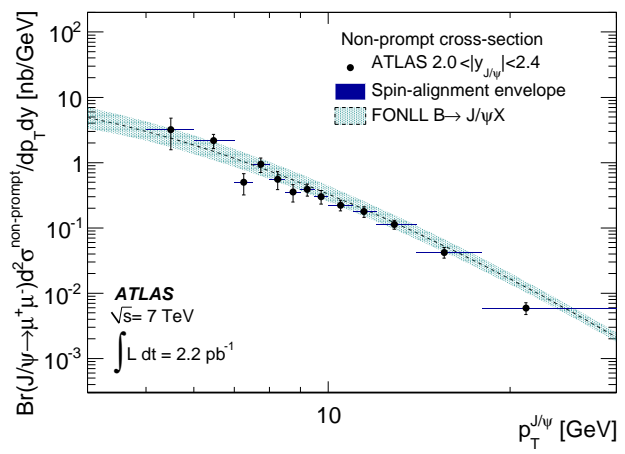

(d) $2<|y|<2.4$

Figure 1: Differential cross section of non-prompt $J / \psi$ in four regions of rapidity for data and theoretical predictions of FONLL. (Plots from [2])

alignment measurements are needed to test existing models. Here the first results of ATLAS concerning $J / \psi$ and $\Upsilon$ production are presented and compared to theoretical models [2,3]. Production of $b$-jets is an important ingredient to understand the $b \bar{b}$ background in searches for new physics. Latest ATLAS published result regarding $b$ and $b \bar{b}$ production using the full 2010 data sample [4] is also presented. For additional information on technical details on these measurement, refer to other talk of this conference [5] on jet reconstruction and $b$-tagging performance in ATLAS.

\subsection{Charm and beauty production via observed $J / \psi$}

At the LHC, $J / \psi$ can be produced either directly from $p$ - $p$ collisions (prompt production, feeddown decays from higher charmonium states like $\chi_{c}, \psi(2 \mathrm{~S})$ are also included) or indirectly from $B$-decays (non-prompt production). By measuring the inclusive $J / \psi$ production and the fraction of non-prompt $J / \psi$, two interesting cross sections are extracted, those of prompt and non-prompt $J / \psi$ production: $\sigma(p p \rightarrow J / \psi+X)_{\text {prompt }}$ and $\sigma\left(p p \rightarrow B+X^{\prime} \rightarrow J / \psi+X^{\prime \prime}\right)$.

In Figure 1 the measured differential cross section of non-prompt $J / \psi$ is shown in four bins of rapidity. A very good description of the measured cross section is provided by FONLL in the $p_{T}$ range from $2 \mathrm{GeV}$ up to $70 \mathrm{GeV}$. The total integrated cross section of non-promt $J / \psi$ is given in two fiducial regions. For $J / \psi$ mesons produced within $|y|<2.4$ and $p_{T}>7 \mathrm{GeV}$, ATLAS provides 


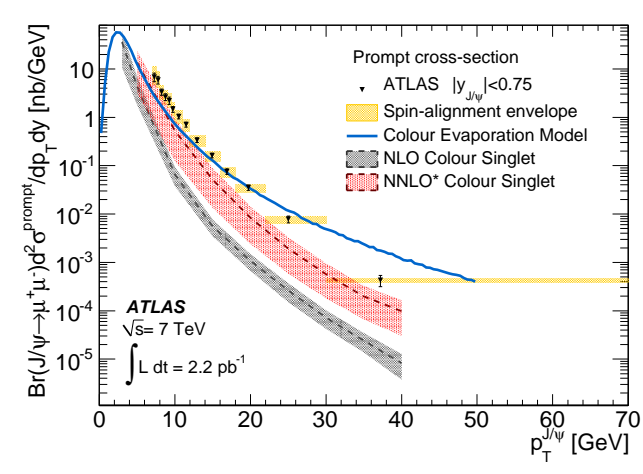

(a) $|y|<0.75$

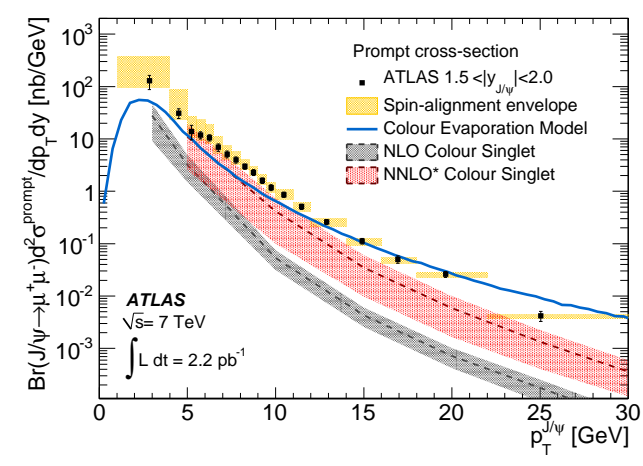

(c) $1.5<|y|<2$

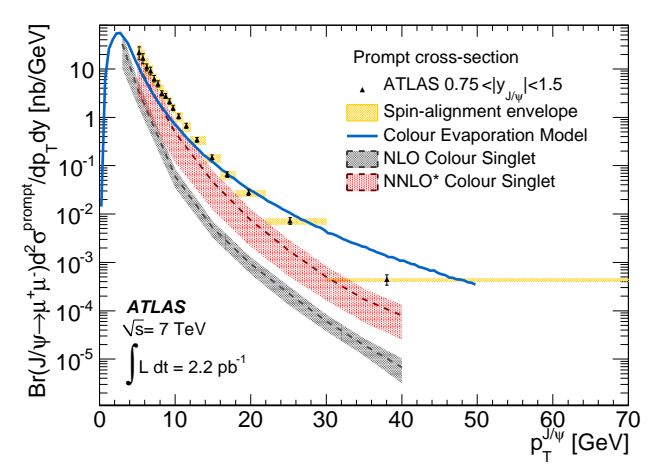

(b) $0.75<|y|<1.5$

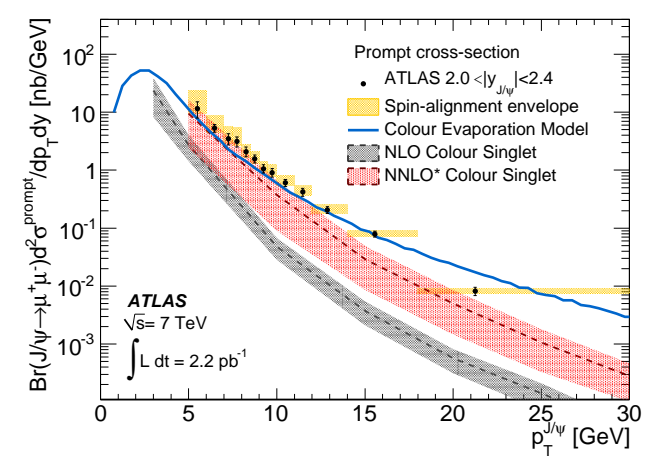

(d) $2<|y|<2.4$

Figure 2: Differential cross section of prompt $J / \psi$ in four regions of rapidity for data and theoretical predictions of Colour Evaporation and Colour Singlet models. (Plots from [2])

a measurement of $13 \%$ total uncertainty:

$$
\begin{aligned}
B r\left(J / \psi \rightarrow \mu^{+} \mu^{-}\right) \sigma(p p & \left.\rightarrow B+X \rightarrow J / \psi X ;\left|y_{J / \psi}\right|<2.4, p_{T}^{J / \psi}>7 \mathrm{GeV}\right) \\
& =23.0 \pm 0.6 \text { (stat.) } \pm 2.8 \text { (syst.) } \pm 0.2 \text { (spin) } \pm 0.8 \text { (lumi.) nb }
\end{aligned}
$$

and for $J / \psi$ mesons produced within $1.5<|y|<2$, ATLAS provides a measurement including $J / \psi$ of low $p_{T}$ down to $1 \mathrm{GeV}$ :

$$
\begin{aligned}
B r\left(J / \psi \rightarrow \mu^{+} \mu^{-}\right) \sigma(p p & \left.\rightarrow B+X \rightarrow J / \psi X ; 1.5<\left|y_{J / \psi}\right|<2, p_{T}^{J / \psi}>1 \mathrm{GeV}\right) \\
& =61 \pm 24 \text { (stat.) } \pm 19 \text { (syst.) } \pm 1 \text { (spin) } \pm 2 \text { (lumi.) nb. }
\end{aligned}
$$

In Figure 2 the measured differential cross section of prompt $J / \psi$ is shown in four bins of rapidity, along with the theoretical predictions of different models. The Colour Evaporation Model (CEM) provides a reasonable prediction of total cross section and an adequate description of the shape in $p_{T}$, given the simplicity of this model. The Colour Singlet Model (CSM) at the next-toleading order (NLO) calculation succeeds in describing the $p_{T}$-shape of the cross section but is low in absolute scale compared to the data. It improves significanlty, however, when adding more orders to the calculation (NNLO*). 


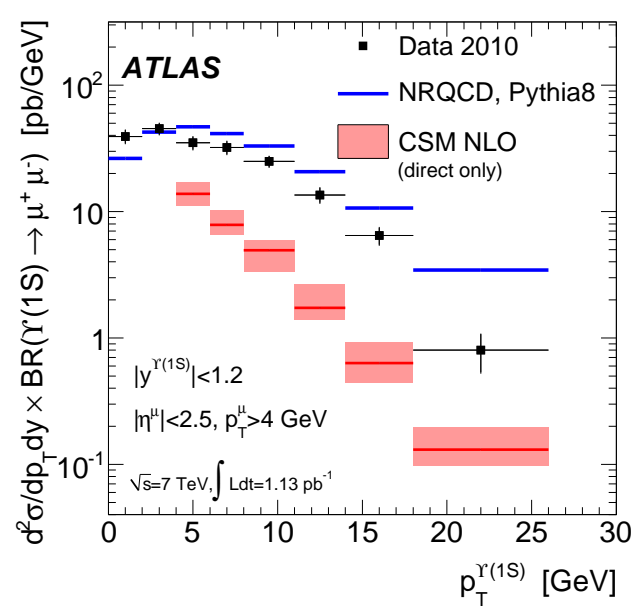

(a) $|y|<0.75$

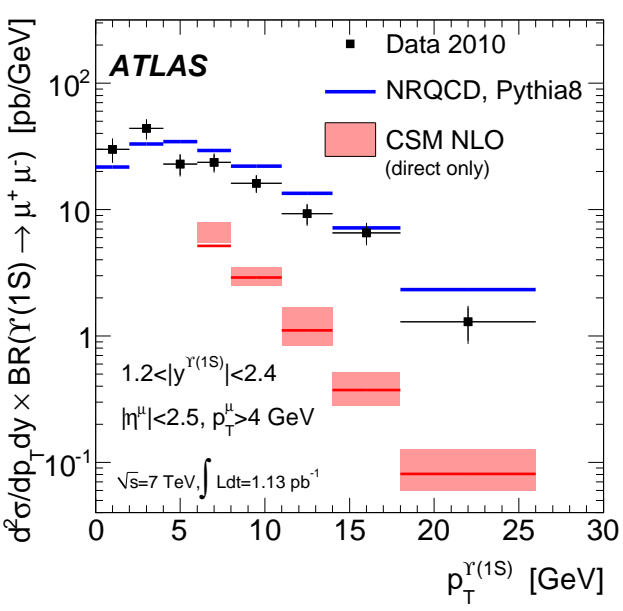

(b) $0.75<|y|<1.5$

Figure 3: Inclusive cross section of $\Upsilon(1 \mathrm{~S})$ in two regions of rapidity for data and theoretical predictions of NRQCD and CSM NLO (only for direct production). (Plots from [3])

The measured total integrated cross section of promt $J / \psi$ is given again in two fiducial regions, one with total uncertainty of $21 \%$ and one including low- $p_{T} J / \psi$ :

$$
\begin{aligned}
B r\left(J / \psi \rightarrow \mu^{+} \mu^{-}\right) \sigma(p p & \left.\rightarrow J / \psi X ;\left|y_{J / \psi}\right|<2.4, p_{T}^{J / \psi}>7 \mathrm{GeV}\right) \\
& =81 \pm 1 \text { (stat.) } \pm 10 \text { (syst.) } \pm_{20}^{25} \text { (spin) } \pm 3 \text { (lumi.) nb } \\
B r\left(J / \psi \rightarrow \mu^{+} \mu^{-}\right) \sigma(p p & \left.\rightarrow J / \psi X ; 1.5<\left|y_{J / \psi}\right|<2, p_{T}^{J / \psi}>1 \mathrm{GeV}\right) \\
= & 510 \pm 70 \text { (stat.) } \pm_{120}^{80} \text { (syst.) } \pm_{130}^{920} \text { (spin) } \pm 20 \text { (lumi.) nb. }
\end{aligned}
$$

\subsection{Bottomonium production via observed $\Upsilon(1 \mathrm{~S})$}

In Figure 3 the measured inclusive differential cross section of $\Upsilon(1 \mathrm{~S})$ is shown in two rapidity bins, $|y|<1.2$ and $1.2<|y|<2.4$. The result is compared to theoretical prediction of Pythia's implemention of non-relativistic QCD (NRQCD) and CSM at NLO. The data show significant deviations from both Monte Carlo (MC) predictions. The CSM NLO calculation is expected to provide a better description of the $p_{T}$-shape of the differential cross section, although here it accounts only for direct production of $\Upsilon(1 \mathrm{~S})$ mesons and does not include any feed-down from excited states ${ }^{1}$.

\subsection{Inclusive $b$-production via $b$-tagged jets}

In Figure 4, the overall picture concerning the production of $b$-jets at LHC is given. ATLAS reports results in four regions of rapidity up to $|y|<2.1$ and in a wide range of $p_{T}$ from 20 to $400 \mathrm{GeV}$, comparing with NLO calculations provided by MC@NLO and POWHEG. In total, the NLO predictions are in good agreement with data. Pythia's prediction for the cross section at leading order is found to be higher by a factor of 1.5 and the $p_{T}$-dependance is well described.

\footnotetext{
${ }^{1}$ In Tevatron, such contributions increase the cross section by a factor of two.
} 


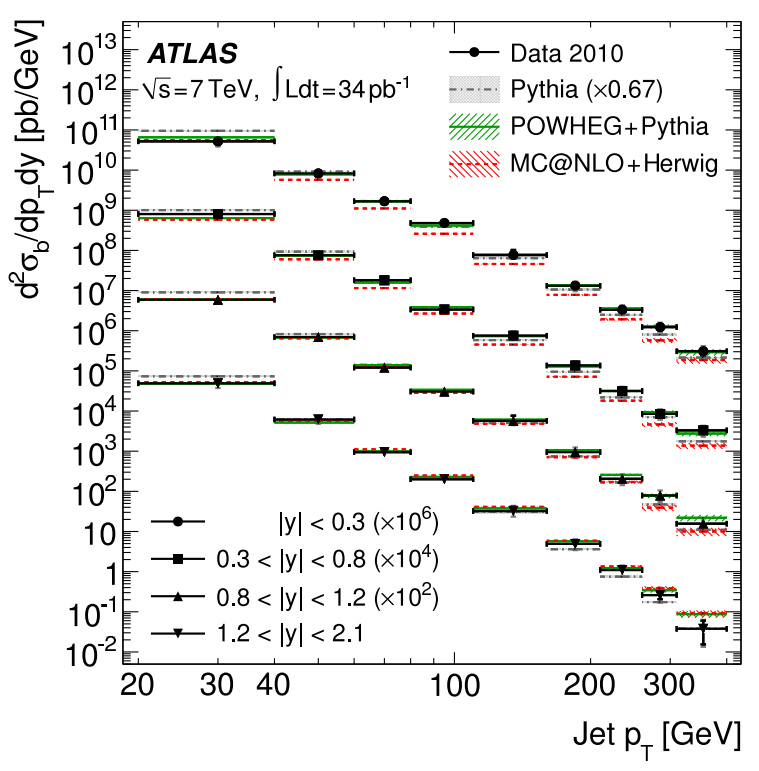

Figure 4: Inclusive double differential $b$-jet cross section as a function of $p_{T}$ for different rapidity ranges compared to predictions of Pythia, POWHEG and MC@ NLO. (Plot from [4])

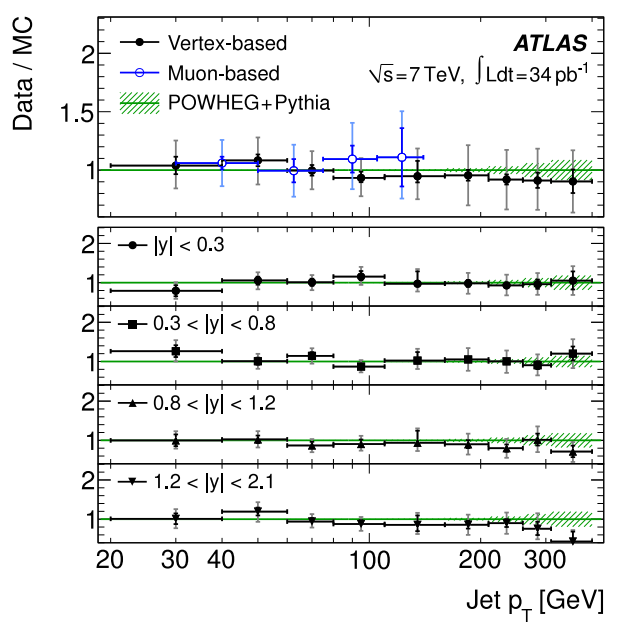

(a)

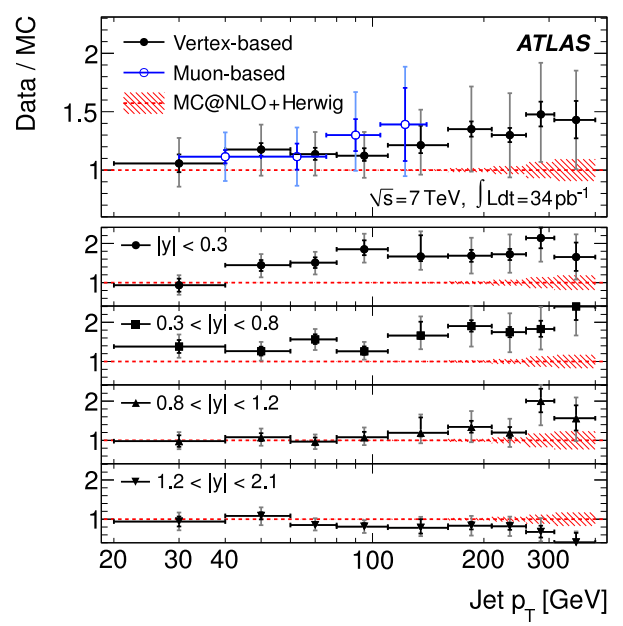

(b)

Figure 5: Ratio of the measured $b$-jet cross sections to the theory predictions of (a) POWHEG and (b) MC@NLO. The top plots show the full rapidity acceptance, while the four smaller plots show the comparison for each of the rapidity ranges separately. (Plots from [4])

To reveal possible differences between data and MC, the ratio between the measured cross section and the NLO prediction of POWHEG and MC@NLO in each $p_{T}$-bin is given in Figure 5. For POWHEG, there seems to be a very good agreement with data not only for the total cross section in the entire $y$-coverage region (top plot), but also in the 4 rapidity bins. MC@NLO predicts different behaviour at high $p_{T}$ when looking at the 4 rapidity bins seperately, but when looking at the cross 


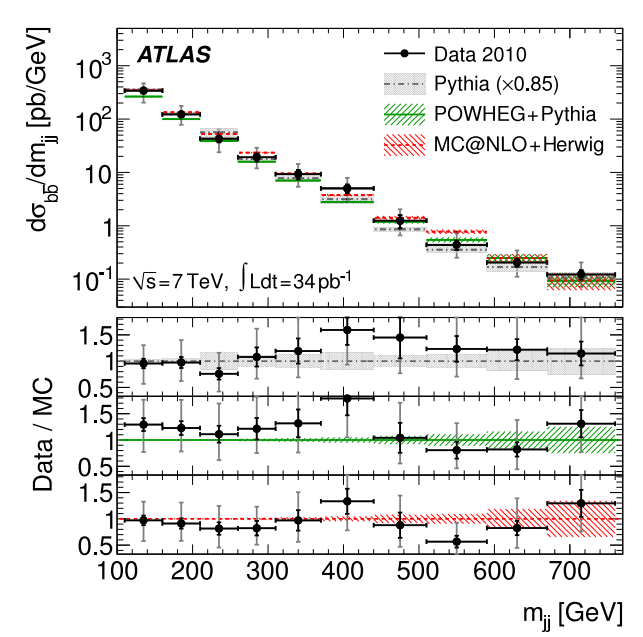

(a)

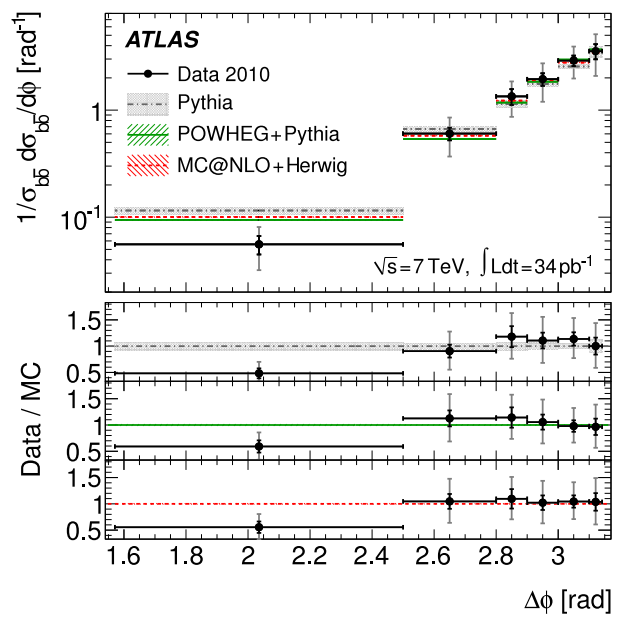

(b)

Figure 6: The $b \bar{b}$ di-jet cross section as a function of di-jet (a) invariant mass and (b) azimuthal angle difference $(\Delta \phi)$ for $b$-jets with $p_{T}>40 \mathrm{GeV}$ and $|y|<2.1$. The data are compared to the MC predictions of Pythia, POWHEG and MC@NLO. (Plots from [4])

section integrated in $y$ the effects from the different $y$-bins seem to cancel out giving a prediction that is consistent with the data, within the high systematic uncertainty of the measurement.

An alternative method to select $b$-jets to the one used as baseline (which is a method searching for a displaced vertex inside jet) was used to validate the cross section measurements. In top plots of Figure 5, the agreement of the two methods in demonstrated in the region where the results of the two methods overlap.

\subsection{Inclusive $b \bar{b}$ di-jet production}

ATLAS has provided results of inclusive $b \bar{b}$ production as a function of various observables in order to disentangle different effects contributing in the production mechanism.

In Figure 6(a) it can be seen that all MC generators provide good description the the di-jet invariant mass spectrum. In Figure 6(b), the $b \bar{b}$ production is shown in bins of difference in azimuthal angle $(\Delta \phi)$ of the di-jet. The enhanced back-to-back behaviour of $b \bar{b}$-production is well reproduced by all generators. At small values of $\Delta \phi$, however, where gluon splitting becomes significant with respect to the other production mechanisms, all MC deviate from the data. In Figure 7, the $\chi=\exp \left(\left|y_{1}\right|-\left|y_{2}\right|\right)$ observable is used in a restricted phase space $\left(\left|y_{j j}^{\text {boost }}\right|=\frac{1}{2}\left|y_{1}+y_{2}\right|<1.1\right)$ in order to perform a test of MC predictions which is relatively insensitive to PDF uncertainties. Again, reasonable agreement between data and $\mathrm{MC}$ is seen.

\section{4. $B$-hadron lifetimes}

Precise measurements of $B$-lifetimes allow tests of theoretical predictions made within the Heavy Quark Expansion framework for the ratios of lifetimes of different $B$-hadron species at per cent level accuracy. In addition, the measurement of the lifetime difference of the two mass eigenstates of the $B_{s}^{0}$ system is of particular interest as it allows the measurement of the $B_{s}^{0}$ mixing 


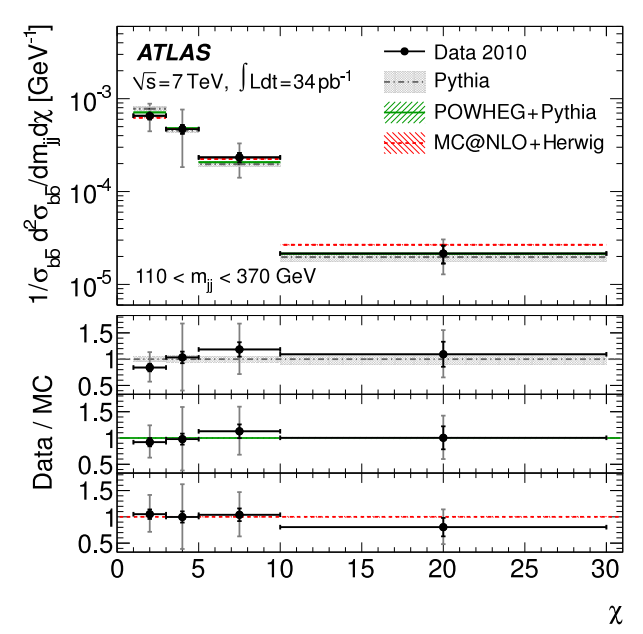

(a) $110<m_{j j}<370 \mathrm{GeV}$

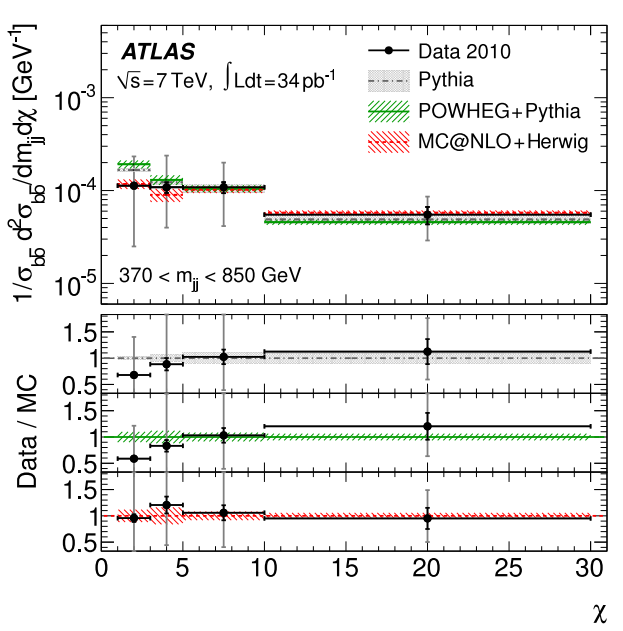

(b) $370<m_{j j}<850 \mathrm{GeV}$

Figure 7: The $b \bar{b}$ di-jet cross section as a function of $\chi$ for $b$-jets with $p_{T}>40 \mathrm{GeV}$ and $|y|<2.1$ in two invariant mass bins. The data are compared to the MC predictions of Pythia, POWHEG and MC@NLO. (Plots from [4])
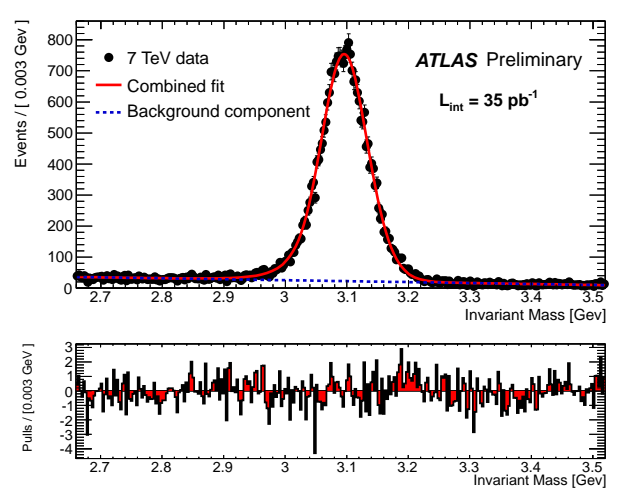

(a)

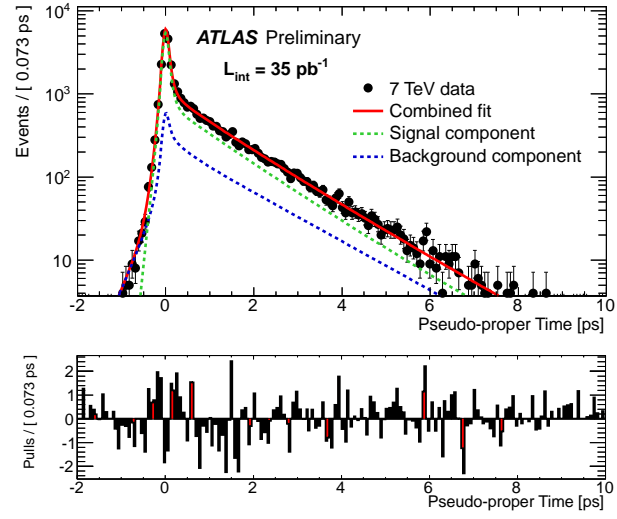

(b)

Figure 8: Invariant mass (a) and pseudo-proper decay time (b) distributions for $B \rightarrow J / \psi+X$ decay candidates. The continuous line is the projection a simultaneous unbinned maximum likelihood fit to the data. The bottom plots are the pull distributions of the fit. (Plots from [6])

phase which generates CP violation in the channel $B_{s}^{0} \rightarrow J / \psi \phi$. The Standard Model predicts that this $\mathrm{CP}$ violating phase is small, thus any measured excess would be a clear indication of new physics. Towards such promising measurements, tracking and secondary vertex reconstruction (decay length resolution, alignment, calibration) have to be validated and that is done with lifetime measurements of inclusive and exclusive $B \rightarrow J / \psi\left(\mu^{+} \mu^{-}\right)+X$ decays. ATLAS measurements are consistent with expectations from world-averaged values [6][7].

An average lifetime measurement of the inclusive sample of $B \rightarrow J / \psi+X$ decays allows detailed studies as it offers higher statistics by orders of magnitude with respect to exclusive $B$ decay channels. This inclusive lifetime measurement gives the average lifetime of the admixture 


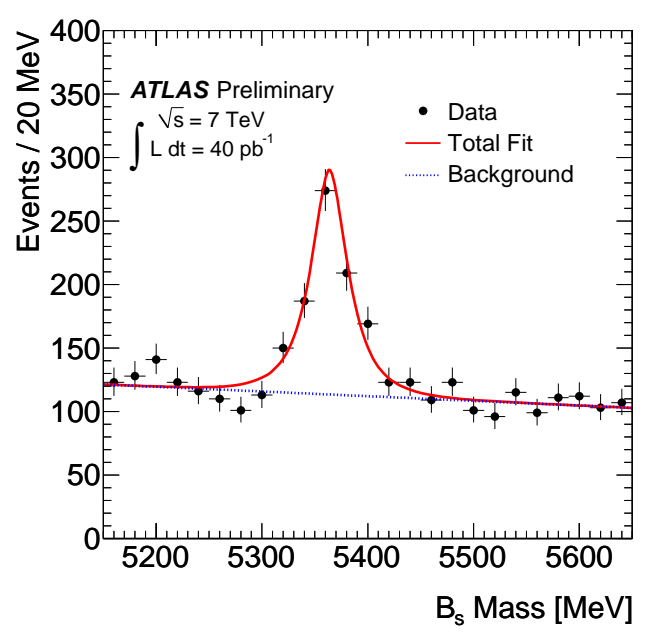

(a)

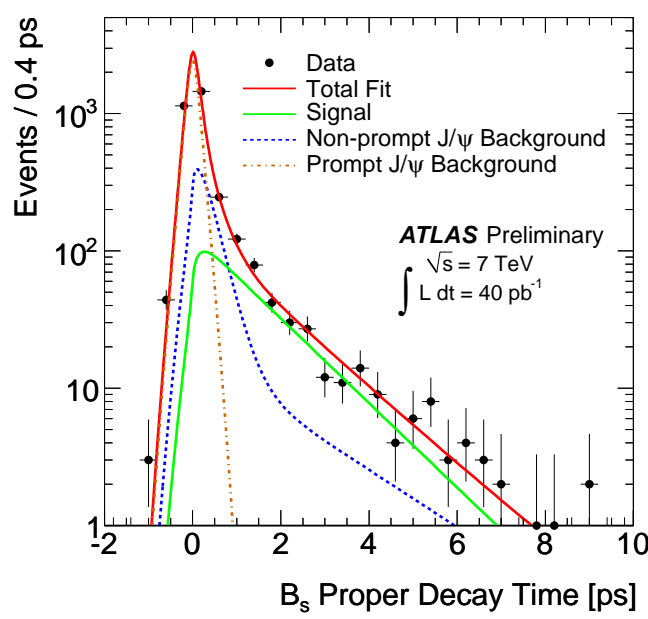

(b)

Figure 9: Invariant mass (a) and pseudo-proper decay time (b) distributions for $B_{s}^{0} \rightarrow J / \psi \phi$ decay candidates. The continuous line is the projection a simultaneous unbinned maximum likelihood fit to the data. (Plots from [7])

of $B$-hadrons decaying to final states including a $J / \psi$ meson. Prompt $J / \psi$ and other di-muon backgrounds are distinguished from non-prompt $J / \psi$ using an unbinned 2D maximum likelihood fit to the invariant mass and pseudo-proper decay time of the di-muon vertex. The result of the fit to the data can be seen in Figure 8. ATLAS result for the average $B$-lifetime is:

$$
\left\langle\tau_{B}\right\rangle=1.1489 \pm 0.016 \text { (stat.) } \pm 0.043 \text { (syst.) ps }
$$

which is found to be in agreement with the most recent measurement provided by the CDF experiment and the expected average lifetime computed using world-averaged values for lifetimes and branching fractions of different $B$-hadron species.

To validate the calibration of the ATLAS tracking system, complementary measurements of exclusive lifetimes are performed using the $B_{d}^{0} \rightarrow J / \psi K^{* 0}$ and $B_{s}^{0} \rightarrow J / \psi \phi$ channels. The technique used to extract the lifetimes is the same as the one used in the average $B$-lifetime measurement and as an example, the result of the fit to data is shown for $B_{s}^{0}$ candidates in Figure 9. The full 2010 data sample is used which provides a yield of $\sim 2750 B_{d}^{0}$ and $\sim 460 B_{s}^{0}$ signal events and the results for the lifetimes are:

$$
\begin{aligned}
& \tau_{B_{d}^{0}}=1.51 \pm 0.04 \text { (stat.) } \pm 0.04 \text { (syst.) ps } \\
& \tau_{B_{s}^{0}}=1.41 \pm 0.08 \text { (stat.) } \pm 0.05 \text { (syst.) ps }
\end{aligned}
$$

In all the above lifetime measurements, detector misalignent is the dominant source of systematic uncertainty. With the limited number of $B_{s}^{0}$ candidates reconstructed in the 2010 data sample, the current measurement ignores the lifetime difference of the mass eigenstates. Within the quoted uncertainties, the extracted lifetimes agree well with the world-averaged values. 


\section{Summary and conclusion}

In this conference, the most up-to-date results of ATLAS concerning $b$-production were presented. The first $B \rightarrow J / \psi+X, b$ and $b \bar{b}$ cross section results show good agreement with NLO calculations. ATLAS has also provided new measurements of $J / \psi$ and $\Upsilon$ production cross sections (differential and/or total). Comparing the results with theoretical predictions of various models, significant improvement is seen in the understanding of quarkonia production.

Apart from measuring cross sections, ATLAS has performed studies of $B$-lifetimes, paving the way for precise measurements which allow tests of pertubative QCD and also of the Standard Model prediction for CP violation in the channel $B_{s}^{0} \rightarrow J / \psi \phi$.

\section{Acknowledgments}

This research has been co-financed by the European Union (European Social Fund - ESF) and Greek national funds through the Operational Program Education and Lifelong Learning of the National Strategic Reference Framework (NSRF) - Research Funding Program: Heracleitus II. Investing in knowledge society through the European Social Fund.

\section{References}

[1] ATLAS Collab., Updated Luminosity Determination in pp Collisions at $\sqrt{s}=7$ TeV using the ATLAS Detector, ATLAS-CONF-2011-011, CERN, Geneva, 2011; https://cdsweb.cern.ch/record/1334563.

[2] ATLAS Collab., Measurement of the differential cross-sections of inclusive, prompt and non-prompt $J / \psi$ production in proton-proton collisions at $\sqrt{s}=7$ TeV, Nucl. Phys. B 850, 387 (2011).

[3] ATLAS Collab., Measurement of the $\Upsilon(1 S)$ Production Cross-Section in pp Collisions at $\sqrt{s}=7 \mathrm{TeV}$ in ATLAS, Phys. Lett. B 705, 9 (2011).

[4] ATLAS Collab., Measurement of the inclusive and dijet cross-sections of b-jets in pp collisions at $\sqrt{s}$ = 7 TeV with the ATLAS detector, Eur. Phys. J. C 71, 1846 (2011).

[5] A. Favareto, Charged track reconstruction and b-tagging performance in ATLAS, in these proceedings.

[6] ATLAS Collab., Measurement of the average $B$ lifetime in inclusive $B \rightarrow J / \psi X \rightarrow \mu^{+} \mu^{-} X$ decays with the ATLAS detector, ATLAS-CONF-2011-145, CERN, Geneva, 2011; https://cdsweb.cern.ch/record/1389455.

[7] ATLAS Collab., Measurement of the $B_{d}^{0}$ and $B_{s}^{0}$ lifetimes in the decay modes $B_{d}^{0} \rightarrow J / \psi K^{* 0}$ and $B_{s}^{0} \rightarrow J / \psi \phi$ in ATLAS, ATLAS-CONF-2011-092, CERN, Geneva, 2011;

https://cdsweb.cern.ch/record/1363779. 